\title{
Preliminary Experimental Investigation on CCRF Barrier Discharge in Supersonic Flow
}

\author{
Hao FAN ${ }^{a}$, Bai-Ling ZHANG, Yi-Wen LI ${ }^{\mathrm{b}, *}$, Ling GAO, Yu-Tian WANG, \\ Cheng-Duo DUAN
}

\author{
Plasma-Dynamic Laboratory, Air Force Engineering University, Xi'an, Shaanxi 710038, China \\ afh0209107@163.com, 'lee_yiwen@163.com \\ ${ }^{*}$ Corresponding author
}

Keywords: MHD, Capacitively Coupled Radio-frequency Barrier Discharge, Double-throat Tunnel, Supersonic Flow.

\begin{abstract}
Producing large-scale and uniform plasma in supersonic field is the foundation of researching cold Magnetohydrodynamic (MHD). The experimental system of capacitively coupled radio-frequency (CCRF) barrier discharge based on Ma3.5 double-throat tunnel is established, and the discharges in the condition of static and supersonic flow are investigating respectively. Main conclusions are as follows. Firstly, a stable and continuous supersonic field is generated by wind tunnel, which could guarantee the discharge experiments working reliably. Secondly, the space distribution of plasma changes and the discharge area reduces in the supersonic flow. Thirdly, the diffusion of plasma is restrained by the magnetic field, which leads to the increase of the degree of ionization.
\end{abstract}

\section{Introduction}

MHD presents a tantalizing opportunity to control hypersonic flows without the need for strong shock waves, low combustion efficiency, and thrust decreases sharply, which has broad potential application prospect in hypersonic aircraft [1-4]. MHD involves the interaction of supersonic field, magnetic field and electric field, currently, the research on MHD mainly depends on computational and theoretical analysis due to complex techniques and strict experimental demands of MHD, relevant ground experimental researches are carried out only in a few countries [5].

Producing large-scale and uniform plasma in supersonic field is the foundation of studying cold MHD, thermal ionization and external ionization are two efficient methods to achieve it. As air temperature is too low for thermal ionization $(\mathrm{T}>2500 \mathrm{~K})$ in the hypersonic aircraft, the external ionization is the only choice [6]. Investigation on external ionization has been implemented by different groups, which especially focus on nanosecond pulseionization and microwave lonization [7-9].

Nanosecond pulsedionization is characterized by high density, low power consumption and high ionization efficiency. Princeton University established the system of MHD power generation based on nanosecond pulsedionization. Short-duration, high-repetition-rate, high-voltage pulses ( $2 \mathrm{~ns}, 100$ $\mathrm{kHz}, \sim 5 \mathrm{kV} / \mathrm{cm})$ are used to ionize a cold, Mach $3(600 \mathrm{~m} / \mathrm{sec}), 0.04 \mathrm{~kg} / \mathrm{m} 3 \mathrm{flow}$, the large-scale and uniform plasma generated in the supersonic flow[10].

Alabama University carried out the experiment investigation on microwave ionization. The plasma is generated by microwave breakdown of the working gas in the MW resonant chamber. The gas exits the resonant chamber and expands through a supersonic nozzle, a supersonic conductive gas attained at the inlet of the MHD channel [11].

However, nanosecond pulsedionization cannot realize the continuous existence of plasma in time domain because of the short half-life of discharge plasma in the air, and the space distribution of microwave plasma is not uniform in the supersonic flow. Those drawbacks will influence the result of the experimental research. In this paper, experimental system of CCRF barrier discharge is established based on Ma3.5 double-throat tunnel and the characteristics of CCRF discharge is 
investigated.

\section{Experimental Setup}

The schematic of experimental setup is shown in Fig 1, the experimental setup is mainly composed of the following sections: wind tunnel, MHD channel, measurement setup, et al.

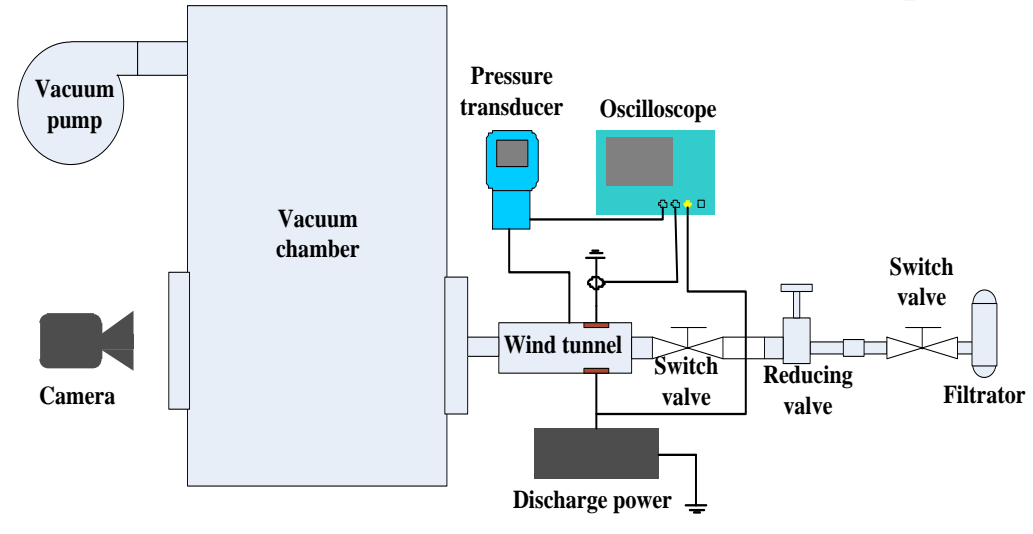

Fig. 1 The Schematic of Experimental Setup

\section{Wind Tunnel}

The molded lines and size of wind tunnel is shown in Fig 2. The double-throat wind tunnel made of organic glass because of its optical clarity, well resistance to abrasion, good electrical insulation, and decent mechanical properties. Considering flow field, magnetic field and electric field comprehensively, the width of test section is designed as $10 \mathrm{~mm}$. The height of cross section of nozzle throat are $2.47 \mathrm{~mm}$, which gives a mass flow rate through the test section of $\mathrm{m}=2.88 \mathrm{~g} / \mathrm{s}$ in design test section condition of $655 \mathrm{~Pa}, 26 \mathrm{~g} / \mathrm{m} 3$ and $87 \mathrm{Kelvin}$ at $654 \mathrm{~m} / \mathrm{s}(\mathrm{P} 0=0.5 \mathrm{~atm})$. The minimum operating inlet/outlet pressure ratio of Ma3.5 double throat is about 4.9, the operating time of wind tunnel is estimated to be 30 s.

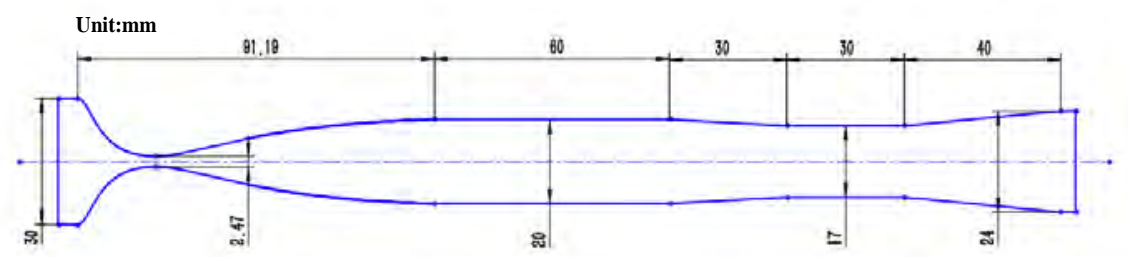

Fig. 2 The Molded Lines and Size of Wind Tunnel

The end of wind tunnel is a vacuum system connected to a $0.8 \mathrm{~m} 3$ vacuum chamber in which air is pumped out by a rotary vane vacuum pump. The minimum pressure in the vacuum system sustained by the pump is $200 \mathrm{~Pa}$. Inlet of wind tunnel is connected to the ambient atmosphere through filtrator, switch valve and reducing valve. Filtrator provides a throughput of air to sweep out impurities and vapor, and reducing valve is designed to adjust the air total pressure ( $\mathrm{P} 0=0.4-1 \mathrm{~atm})$.

Fig 3 shows the static pressure variation in test section during wind tunnel operating $(\mathrm{P} 0=0.5 \mathrm{~atm})$. The static pressure of test section is $650 \mathrm{~Pa}$, during operating time $(18 \mathrm{~s})$. The smooth waveform means that the flow field is steady, which can meet the requirement of MHD steady technical experiment. In the experiment, a shock wave is generated in the downstream of wind tunnel with pressure in vacuum chamber increasing. Shock wave would be pushed forward to test section as the back pressure continues increasing. The static pressure increases suddenly when shock wave reaches test section, which means the end of operation. 


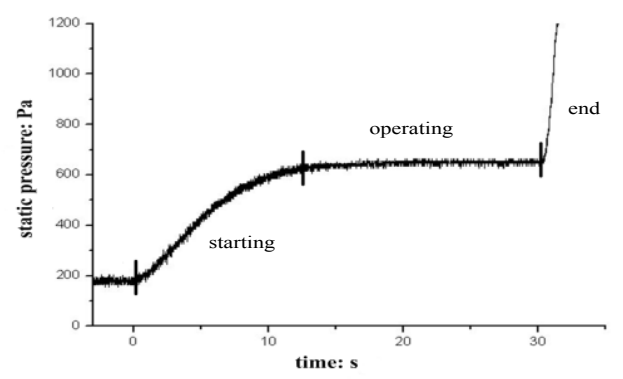

Fig. 3 Static Pressure Variation in Test Channel During Wind Tunnel Operating

\section{Ionization}

In the MHD technical experiment, the gas must be ionized. In this system, conductive gas is obtained by CCRF barrier discharge, during which high output power (up to 200W), and high discharge frequency (10khz-10Mhz) are applied. To inject more power into test section, two inductance coils are used to match the discharge circuit.

Fig 4 shows a schematic of electrode block. Two rectangular electrode block $45 \mathrm{~mm}$ long are flush mounted in the side test section walls. Each electrode, made of alumina ceramic, incorporates a single palladium plate electrode with $16 \mathrm{~mm}$ wide, $20 \mathrm{~mm}$ long. The electrode edges are rounded using a Rogowski profile to achieve a more uniform electric field distribution between the electrodes. $1 \mathrm{~mm}$ thick alumina ceramic is used to prevent generating electrospark and electric arc in the test section, improve the uniformity of conductive gas.

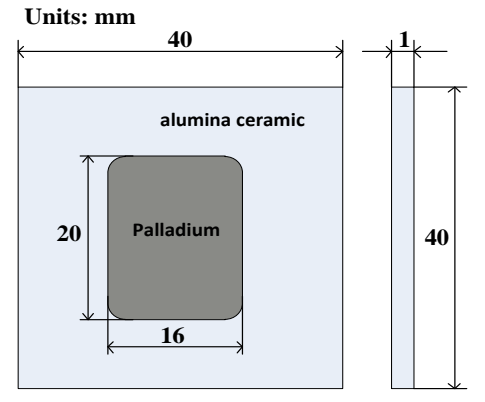

Fig. 4 The Schematic of Electrode Block

\section{Magnet}

The applied magnetic field in this experiment is generated by using anWD-200 DC water cooled electromagnet. Magnetic field is generated between the circular poles with diameter of $90 \mathrm{~mm}$, which is uniform over this test cube. In the experiments, for the $30 \mathrm{~mm}$ between the poles, the magnetic field generated by the magnet coils at a maximum current of $30 \mathrm{~A}$ is $1.8 \mathrm{~T}$.

\section{Measurement Setup}

For the electrical parameters measurement, TekP6015A high voltage probe is used for discharge voltage measurement, and TCPA300+TCP312 current probe is used for discharge current measurement. Both of which are acquired and recorded by DPO4104 oscilloscope. The static pressure in test section is measured with an absolute pressure transducer, and inlet total pressure with a vacuum gauge. Besides, images of discharge in the test section are recorded by a camera.

\section{Preliminary Experimental Investigation of CCRF Barrier Discharge}

\section{Static Condition}

The discharge images with the LP(load power) of $22 \mathrm{~W}$ and pressure varying from $330 \mathrm{~Pa}$ to $1500 \mathrm{~Pa}$ 
are shown in Fig 5, which shows that CCRF discharges have two typical different forms. The ionized plasma is homogenously distributed in the whole test section when $\mathrm{P}=330 \mathrm{~Pa}$, weak-current discharge. The discharge form turns into a strong-current discharge as the pressure increases. At this time, there are three regions in test section including negative glow, Faraday dark space and positive column[12].

Comparative analysis from Fig b, Fig c, Fig d, directly shows that with the increase of pressure, negative glow space become smaller, which positive column space and Faraday dark space turn to be larger, and the average degree of ionization reduces in general. In the case of constant pressure, the average degree of ionization increases with the increase of load power.

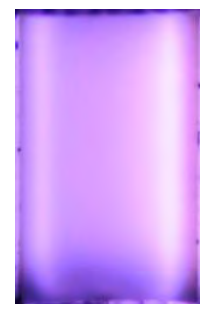

(a) $330 \mathrm{~Pa}$

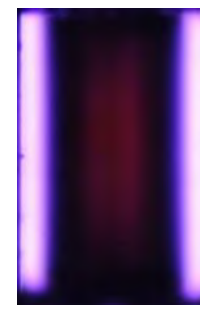

(b) $750 \mathrm{~Pa}$

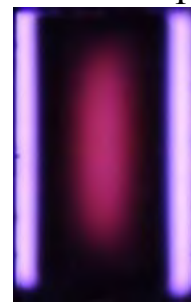

(c) $1000 \mathrm{~Pa}$

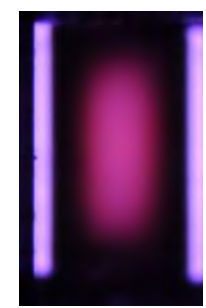

(d) $1500 \mathrm{~Pa}$

Fig. 5 Discharge Images with Different Pressure in Static Air

\section{Magnetic Field}

Fig 6 shows the space distribution of plasma in the magnetic field, shape of positive column change a lot. The discharge plasma drifts along the channel before joining transverse magnetic field. The direction of drift changes with Lorentz force after joining magnetic field, and the discharge plasma get together near the upper and lower wall. Besides, the width of central area is reduced in the positive column space. The diffusion of plasma is restrained in the magnetic field, and the free electron density's increasing lead to the increases of degree of ionization.



$\mathrm{B}=0 \mathrm{~T}$

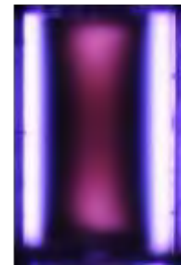

$\mathrm{B}=1.1 \mathrm{~T}$

Fig. 6 Discharge Images in the Magnetic Field

\section{Supersonic Condition}

The images of discharge in static and supersonic flow are shown in Fig 7. Compared with discharge in the static condition, the area of discharge is significantly reduced in the supersonic flow. A large proportion of output power loses along with supersonic flow lead to reduction of discharge area. Besides, the parameters distribute unequally in the supersonic flow gas, which leads to the changing of space distribution of plasma, especially in the boundary layer. Finally, due to the smaller concentration of particles number in the supersonic field in the condition of same pressure, the density of plasma of central area becomes larger.



(a)Static condition

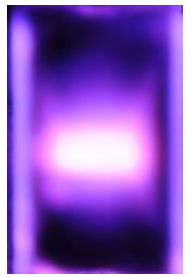

(b)Supersonic Condition

Fig. 7 Discharge Images in Static and Supersonic Flow 


\section{Conclusion}

(1)A stable and continuous supersonic field is generated by wind tunnel, which could guarantee the discharge experiments' work reliably.

(2)Compared with that in static condition, the space distribution of plasma changes and the area of discharge reduce in the supersonic flow.

(3)The diffusion of plasma is restrained in the magnetic field, which leads to the increase of the degree of ionization.

\section{Acknowledgement}

This research was financially supported by the National Natural Science Foundation of China under Grants 51306207and 11372352 and Natural Science Foundation of shaanxi province under Grants 2013JQ1016.

\section{References}

[1]Kuranov A, Korabelnikov A. Atmospheric Cruise Flight Challenges for Hypersonic Vehicles under the AjaxConcept[J]. Journal of Propulsion and Power, 2008, 24(6): 1129.

[2]Kuranov A, Korabelnikov A. Hypersonic technologies of atmospheric cruise flight under AJAX concept[R]. AIAA 2008-2524, 2008.

[3]Sheikin E G, Kuranov A L. Scramjet with MHD Bypass under "Ajax" Concept[R]. AIAA 2004-1192, 2004.

[4]Tang J F, Bao W, Yu D R. A New Manner for Energy Reintroduction in AJAX[R]. AIAA 2006-8101, 2006.

[5]LI Yiwen, LI Yinghong, LU Haoyu, ZHU Tao, ZHANG Bailing. Preliminary experimental investigation on MHD power generation using seeded supersonic argon flow as working fluid[J]. Chinese Journal of Aeronautics, 24(12): 701-708, 2011.

[6]UDAGAWA K, KAWAGUCHI K, SAITO S, et al. Experimental Study on Supersonic Flow Control by MHD Interaction[R]. AIAA 2008-4222, 2008.

[7]Murray R C, Zaidi S H, Shneider M N, et al. Non-equilibrium ionization techniques for MHD power extraction in high-speed flows[R]. AIAA 2003-1049, 2003.

[8]Murray R C, Zaidi S H, Carraro M R, et al. Observation of MHD effects with non-equilibrium ionization in cold supersonic air flows[R]. AIAA 2004-1025, 2004.

[9]McAndrew B, Barker P, Miles R. Development of a Supersonic Plasma Wind Tunnel[C]. 38th Aerospace Sciences Meeting and Exhibit, 2000.

[10]Murray R C, Zaidi S H, Carraro M R, et al. Magnetohydrodynamic Power Generation Using Externally Ionized, Cold, Supersonic Air as Working Fluid[J]. AIAA Journal, 2006, 44(1):119-127.

[11]Deaconu S, Jones J E, Hawk C W, et al. Diagnostics of electric properties of flowing microwave plasmas for MHD applications AIAA-99-2715, 1999.

[12]Raizer Y P. Gas Discharge Physics[M]. Berlin, Germany. Springer-Verlag, 1991. 